University of Vermont

UVM ScholarWorks

College of Arts and Sciences Faculty

Publications

College of Arts and Sciences

2-1-2017

\title{
Estimates of local biodiversity change over time stand up to scrutiny
}

\author{
Mark Vellend \\ Université de Sherbrooke \\ Maria Dornelas \\ University of St Andrews \\ Lander Baeten \\ Universiteit Gent \\ Robin Beauséjour \\ Université de Sherbrooke \\ Carissa D. Brown \\ Memorial University of Newfoundland
}

See next page for additional authors

Follow this and additional works at: https://scholarworks.uvm.edu/casfac

Part of the Climate Commons

\footnotetext{
Recommended Citation

Vellend M, Dornelas M, Baeten L, Beauséjour R, Brown CD, De Frenne P, Elmendorf SC, Gotelli NJ, Moyes F, Myers-Smith IH, Magurran AE. Estimates of local biodiversity change over time stand up to scrutiny. Ecology. 2017 Feb;98(2):583-90.

This Article is brought to you for free and open access by the College of Arts and Sciences at UVM ScholarWorks. It has been accepted for inclusion in College of Arts and Sciences Faculty Publications by an authorized administrator of UVM ScholarWorks. For more information, please contact scholarworks@uvm.edu.
} 


\section{Authors}

Mark Vellend, Maria Dornelas, Lander Baeten, Robin Beauséjour, Carissa D. Brown, Pieter De Frenne, Sarah C. Elmendorf, Nicholas J. Gotelli, Faye Moyes, Isla H. Myers-Smith, Anne E. Magurran, Brian J. McGill, Hideyasu Shimadzu, and Caya Sievers 


\title{
Estimates of local biodiversity change over time stand up to scrutiny
}

\author{
Mark Vellend,, 11 Maria Dornelas, ${ }^{2}$ Lander Baeten, ${ }^{3}$ Robin Beauséjour, ${ }^{1}$ Carissa D. Brown, ${ }^{4}$ \\ Pieter De Frenne, ${ }^{3,5}$ Sarah C. Elmendorf, ${ }^{6}$ Nicholas J. Gotelli, ${ }^{7}$ Faye Moyes, ${ }^{2}$ Isla H. Myers-Smith, ${ }^{8}$ \\ Anne E. Magurran, ${ }^{2}$ Brian J. McGill, ${ }^{9}$ Hideyasu Shimadzu, ${ }^{10}$ and Caya Sievers ${ }^{2}$ \\ ${ }^{1}$ Département de Biologie, Université de Sherbrooke, 2500 boulevard de l'Université, Sherbrooke, Quebec J1K 2 Rl Canada \\ ${ }^{2}$ Centre for Biological Diversity and Scottish Oceans Institute, School of Biology, University of St. Andrews, \\ St. Andrews, Fife, KY16 9 TH United Kingdom \\ ${ }^{3}$ Department of Forest and Water Management, Forest \& Nature Lab, Ghent University, BE-9090, Melle-Gontrode, Belgium \\ ${ }^{4}$ Department of Geography, Memorial University, St. John's, Newfoundland and Labrador A1 B 3 X9 Canada \\ ${ }^{5}$ Department of Plant Production, Ghent University, Proefhoevestraat 22, 9090, Melle, Belgium \\ ${ }^{6}$ National Ecological Observatory Network, Boulder, Colorado 80301 USA \\ ${ }^{7}$ Department of Biology, University of Vermont, Burlington, Vermont 05405 USA \\ ${ }^{8}$ School of GeoSciences, University of Edinburgh, Edinburgh, EH9 3 FF United Kingdom \\ ${ }^{9}$ School of Biology and Ecology, Sustainability Solutions Initiative, University of Maine, Orono, Maine 04469 USA \\ ${ }^{10}$ Department of Mathematical Sciences, Loughborough University, Loughborough, Leicestershire, LE11 $3 T U$ United Kingdom
}

\begin{abstract}
We present new data and analyses revealing fundamental flaws in a critique of two recent meta-analyses of local-scale temporal biodiversity change. First, the conclusion that short-term time series lead to biased estimates of long-term change was based on two errors in the simulations used to support it. Second, the conclusion of negative relationships between temporal biodiversity change and study duration was entirely dependent on unrealistic model assumptions, the use of a subset of data, and inclusion of one outlier data point in one study. Third, the finding of a decline in local biodiversity, after eliminating post-disturbance studies, is not robust to alternative analyses on the original data set, and is absent in a larger, updated data set. Finally, the undebatable point, noted in both original papers, that studies in the ecological literature are geographically biased, was used to cast doubt on the conclusion that, outside of areas converted to croplands or asphalt, the distribution of biodiversity trends is centered approximately on zero. Future studies may modify conclusions, but at present, alternative conclusions based on the geographic-bias argument rely on speculation. In sum, the critique raises points of uncertainty typical of all ecological studies, but does not provide an evidence-based alternative interpretation.
\end{abstract}

Key words: biodiversity; disturbance; geographic bias; meta-analysis; species richness; temporal change; time series.

\section{INTRODUCTION}

Patterns of biodiversity over space and time represent the foundation of many ecological theories and conservation prioritization schemes. Concerns have been raised (Gonzalez et al. 2016) about two papers that collectively analyzed $>250$ individual data sets on biodiversity change through time from many parts of the world (Vellend et al. 2013, Dornelas et al. 2014). Both of these studies found that the average magnitude of temporal change in alpha diversity across studies was indistinguishable from zero. Dornelas et al. (2014) additionally showed significant and consistent temporal species turnover, thus highlighting marked shifts in community composition. The concerns

Manuscript received 25 July 2016; revised 14 October 2016; accepted 8 November 2016. Corresponding Editor: Helmut Hillebrand.

${ }^{11}$ E-mail: mark.vellend@usherbrooke.ca of Gonzalez et al., which focus on species richness and diversity, are for the most part typical of those that could be directed at any ecological meta-analysis: different results might obtain in different places (underrepresented regions) or times (before people collected data of this nature), and it is possible to find data subsets that deviate from the overall pattern. These concerns were used by Gonzalez et al. to call into question our conclusions. Some aspects of the Gonzalez et al. critique relied on their selective use of data and methods of analysis, while others focused on the nature of the data themselves and accompanying interpretations.

Here we present analyses, as well as new data, to support the following conclusions: (1) Well-replicated short-term time series do not provide biased estimates of long-term biodiversity trends. The opposite conclusion presented by Gonzalez et al. was based on two errors in their simulation model and calculations. (2) There is no 
compelling evidence that studies of longer temporal duration show greater biodiversity decline. On this point, the analyses presented by Gonzalez et al. were contingent on a single outlier data point and to unrealistic assumptions about model structure, and the results were not robust to the inclusion of additional data. (3) There is no evidence in our original analyses or using a larger, updated data set that the results were biased due to combining the effects of disturbance with post-disturbance dynamics. In fact, selectively excluding post-disturbance dynamics is itself a source of bias. (4) The ecological literature is indeed geographically biased, a fact discussed explicitly in both Vellend et al. (2013) and Dornelas et al. (2014). The analysis of Gonzalez et al. on this issue supports the undebatable conclusion that new data (in this case from underrepresented regions) might modify conclusions from these meta-analyses, or from any other global-scale ecological meta-analysis.

\section{Short-Term Time Series Do Not Provide Biased Estimates OF LONG-Term TRENDS}

As we show below, a key component of the Gonzalez et al. critique is incorrect (i.e., not a matter of selective interpretation). Simulations of species richness $(S)$ over 50 -yr periods and subsequent calculations of $\log$ ratios $\left(\log \left(S_{\text {after }} / S_{\text {before }}\right)\right)$ or slopes of richness on time during shorter time intervals $(5,10,20 \mathrm{yr})$ were used to argue that "Estimates of biodiversity change are systematically biased when syntheses are based on data sets composed primarily of short time series." Gonzalez et al. made two different errors, the first of which applies only to log ratios, the second of which applies to both log ratios and slopes:

First, when calculating a mean effect size for "short" windows of time, Gonzalez et al. did not take into account the fact that a log ratio across, for example, a 10-yr period is only expected (mathematically) to capture one-fifth of the amount of change that occurs over $50 \mathrm{yr}$. In other words, they did not multiply the average of 10 -yr windows by 5 before comparing with the 50 -yr effect size. This is equivalent to the argument that, hypothetically, temperature only went up by $0.5^{\circ} \mathrm{C}$ per decade, so the estimate of the "real" increase of $2.5^{\circ} \mathrm{C}$ over 50 years is biased.

The second problem is less obvious, but no less important, and it accounts for apparent diversity increases in medium-sized time windows (e.g., $20 \mathrm{yr}$ ) when a 50-yr period shows a richness decline initially, followed by an increase, and then a leveling off (see Fig. 1a-c). The problem is that with a bounded range of $50 \mathrm{yr}$, "randomly" chosen segments of $20 \mathrm{yr}$ severely over-represent the middle portion of the time series. In another well-known ecological context, this is called the mid-domain effect to explain peak species richness at central latitudes or altitudes (Colwell and Lees 2000). However, whereas the boundaries in space are real, the temporal boundaries are not, as time may be considered infinite in both directions. The first point in the time series, for example, is only part of one 20-yr segment in the "population" from which the Gonzalez et al. simulations sample, $0-20$. The second time point is part of two segments, 0-20 and 1-21, and so on. Time points 20-30, on the other hand, are each part of 20 different segments. So, with the decline in richness happening early during the 50-yr time span, seemingly random samples of $20 \mathrm{yr}$ mostly miss the decline, while "detecting" a transient increase only because it happens to occur in the middle portion of the time series. The apparent bias detected by Gonzalez et al. is an artefact of their simulation analysis focusing on an arbitrary bounded time interval (Fig. 1).

If one examines sequential, non-overlapping portions of any length of a given time series, the average log ratio captures precisely the rate of change over the entire time series. Simulations are not required to demonstrate this point, although we provide one corrected example from Gonzalez et al. (Fig. 1), in addition to the following explanation from first principles. Imagine we have a species richness $(S)$ time series of five points, $t_{0}: t_{4}$, and thus four year-to-year transitions. The log ratio from beginning to end is $\log \left(S_{4} / S_{0}\right)$. The average of $1-y r$ intervals is:

$$
\begin{aligned}
= & \left(\log \left(\mathrm{S}_{1} / \mathrm{S}_{0}\right)+\log \left(\mathrm{S}_{2} / \mathrm{S}_{1}\right)+\log \left(\mathrm{S}_{3} / \mathrm{S}_{2}\right)+\log \left(\mathrm{S}_{4} / \mathrm{S}_{3}\right)\right) / 4 \\
= & \left(\log \left(\mathrm{S}_{1}\right)-\log \left(\mathrm{S}_{0}\right)+\log \left(\mathrm{S}_{2}\right)-\log \left(\mathrm{S}_{1}\right)+\log \left(\mathrm{S}_{3}\right)\right. \\
& \left.-\log \left(\mathrm{S}_{2}\right)+\log \left(\mathrm{S}_{4}\right)-\log \left(\mathrm{S}_{3}\right)\right) / 4 \\
= & \left(\log \left(\mathrm{S}_{4}\right)-\log \left(\mathrm{S}_{0}\right)\right) / 4 \\
= & \log \left(\mathrm{S}_{4} / \mathrm{S}_{0}\right) / 4
\end{aligned}
$$

So, as long as we account for the fact that the 1-yr intervals cover only one-quarter of the full time series (i.e., we multiply this by four), we recover the original "target" $\log$ ratio for the full time series precisely (see also Fig. 1g). The same result will hold for 2-yr intervals in this time series, 10-yr intervals of a 50-yr time series, or any other combination. The same precise mathematical equivalence does not hold for slopes, but it is equally true that there is no systematic bias introduced by the fact of sampling a subset of a longer time series. An incomplete sample of the portions of the longer time series will introduce variance (as is always the case with sampling), but not systematic bias (Fig. 1). The conclusion, based on simulations, "that short time series can provide unreliable estimates of a known trend" (Gonzalez et al. 2016) is simply incorrect.

\section{Local Biodiversity Trends in Studies of Different Duration}

The argument that short-term time series bias estimates of temporal biodiversity trends was used by Gonzalez et al. as a springboard to asking whether longer duration studies tend to show biodiversity declines. In this section, we address this issue for the two original studies in turn. 
a) 50 -yr species richness time series

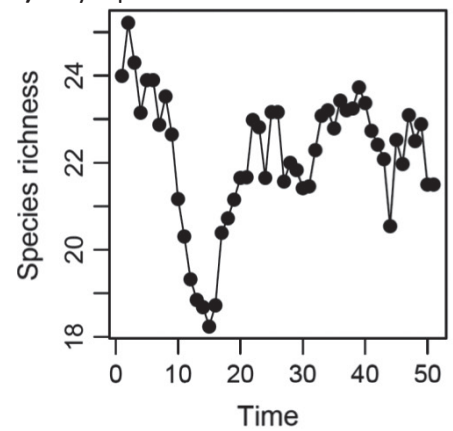

d) LR: corrected for duration

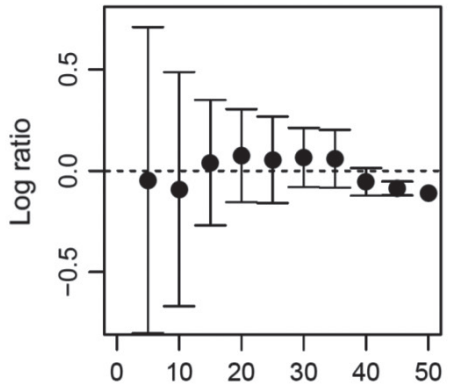

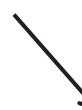

b) Gonzalez et al. results: log ratios (LR)
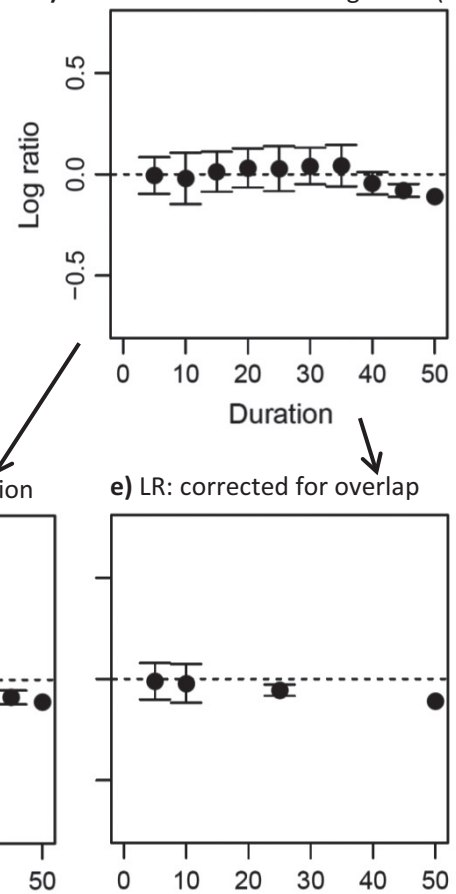

g) LR: corrected for duration and overlap

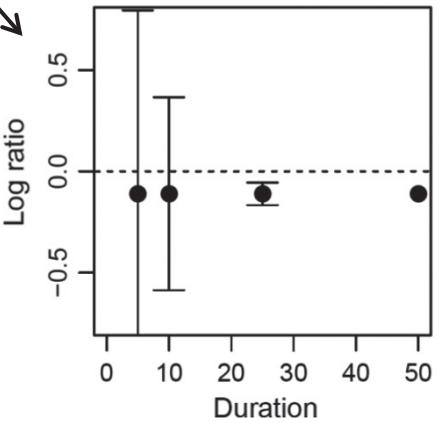

c) Gonzalez et al. results: slopes
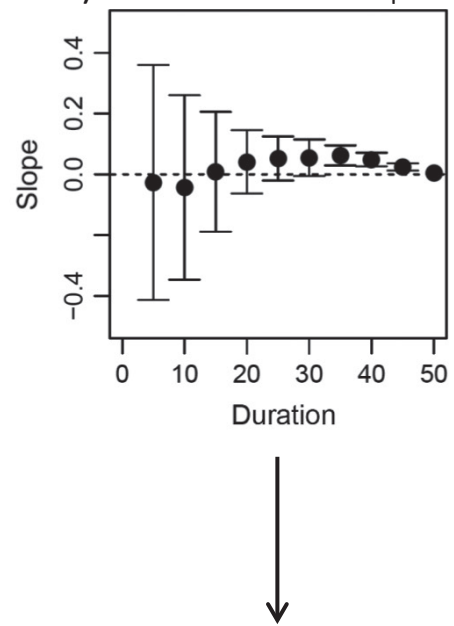

f) Slopes: corrected for overlap

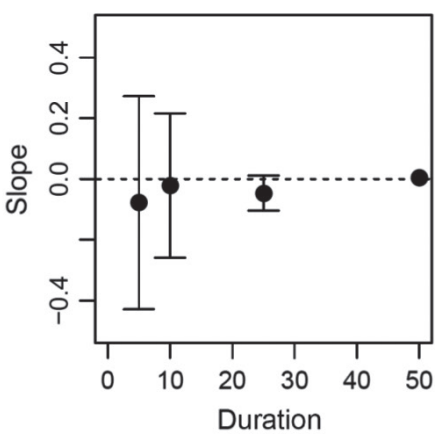

FIG. 1. Log ratios of species richness, $S\left(\log \left(S_{\text {after }} / S_{\text {before }}\right)\right.$ ), and slopes (species richness vs. time) for repeated samples of short time series sampled from a longer (50-yr) duration data set. Values are mean \pm SD. (a) A reproduction of Fig. S3D from Gonzalez et al., showing one example of species richness dynamics over time that appeared to lead to especially biased results. (b, c) Results of 1,000 seemingly random samples of different duration conducted according to the methods of Gonzalez et al.; these results appear to show an average positive trend among moderate-duration samples, despite a long-term negative (log ratio) or flat (slope) trend over the full duration. (d, e) Log ratio results when correcting separately for duration (problem 1 in Short-Term Time Series Do Not Provide Biased Estimates of Long-Term Trends) and overlap (problem 2); here we see that just accounting for the duration of data subsets removes bias from short-duration samples, while correcting for overlap removes any tendency for positive average trends. (f) Slope results after correcting the overlap problem. (g) Log ratio results after correcting for both problems; here the averages are precisely equal to the long-term trend. Note that when correcting for overlap, we only use durations that are multiples of the 50 -yr total time span.

Using the data from Vellend et al. (2013), Gonzalez et al. modeled the log ratio of species richness at the end and start of a study (see Short-Term Time Series Do Not Provide Biased Estimates of Long-Term Trends) as a function of the duration of that study, finding a statistically significant $(P=0.04)$ but weak relationship (Fig. 2a). They emphasized the conclusion that longer-duration studies tend to show richness declines, although by allowing for a non-zero intercept, their results also require explaining a nonsensical positive biodiversity trend in studies that last zero years. If one makes the ecologically realistic assumption that the log ratio must be zero at duration $=0$ (i.e., a zero intercept), not only is the slope not significant, but its raw value is actually positive rather than negative (Fig. 2b). This illustrates the potentially major influence of assumptions about model structure on the spurious detection of weak statistical relationships. 


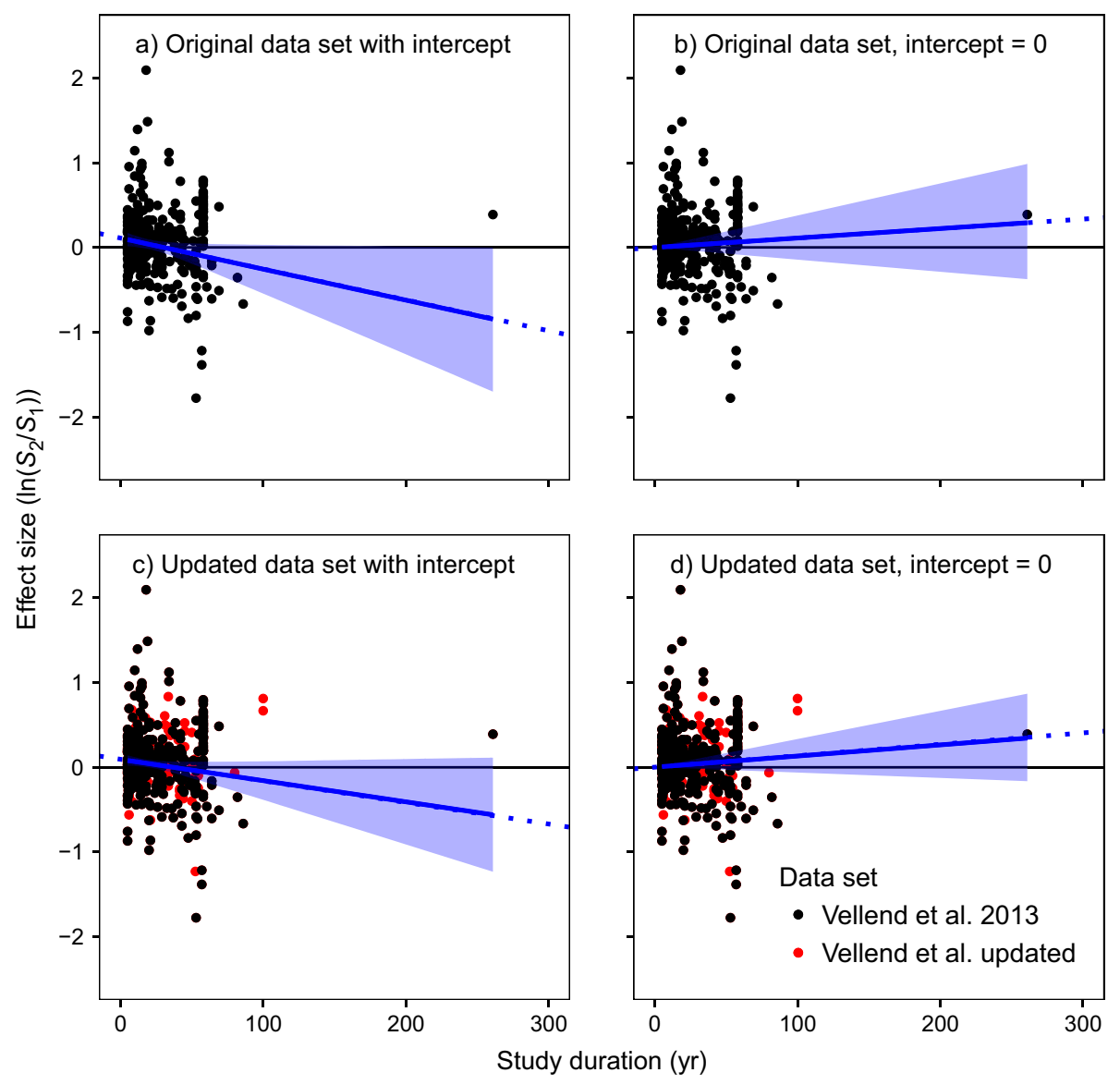

FIG. 2. Relationships between local plant species richness change over time ( $y$-axis) and the duration of a study, modeled assuming either (a, c) a non-zero $y$-intercept or (b, d) a zero $y$-intercept, using (a, b) the original data in Vellend et al. (2013) or (c, d) an expanded data set (c, d; new data points shown in red). The effect size for temporal richness change is expressed as the log ratio of species richness in the final year of study $\left(\mathrm{S}_{2}\right)$ and in the initial year of study $\left(\mathrm{S}_{1}\right)$. Lines represent the estimated effect size with credible intervals. See Appendix S1 for statistical methods and Metadata S1 for all data and computer code. [Color figure can be viewed at wileyonlinelibrary.com]

Given the controversy sparked by Vellend et al. (2013), we have since expanded the data set by $37 \%$ to include studies published through the end of 2014 (the original paper had studies published up to July 2012; see Metadata S1 for data and computer code). The methods were identical to those in Vellend et al. (2013), except that we did not additionally read through the references of all new papers to find additional data sets. With the larger data set of 212 studies (the 2013 paper had 155), there is no significant relationship between local richness change and study duration, regardless of whether one allows for a non-zero intercept (Fig. 2c, d).

The data in Dornelas et al. (2014) include studies with diversity estimates for at least three time points, thus allowing the estimation of slopes of diversity vs. time, rather than only before-after log ratios. There is no significant relationship between the diversity-time slope and study duration (Fig. 3a, b). Gonzalez et al. chose instead to calculate log ratios using the data in Dornelas et al.
(2014; see Data set S1 in that paper), thereby excluding most of the data used by Dornelas et al., and reported a significant negative relationship between log ratios and study duration (Fig. 3c). Again their analysis allowed for a non-zero intercept; if the intercept is fixed at zero, as expected after no time has elapsed, the relationship is not significant (Fig. 3d). In addition, the Gonzalez et al. result is highly sensitive to one outlier, depending not just on a single study (reference 90 in Dornelas et al. 2014), but on a single data point in that study (species richness $=43$ in 1911 , and $<20$ for the next 90 years). In the absence of that one data point, the relationship is not statistically significant, regardless of whether one assumes a zero or non-zero intercept (Fig. 3e, f).

In sum, the evidence provided by Gonzalez et al. to support their claim that longer-duration studies tend to show biodiversity decline is exceedingly weak at best. Their conclusions depend on specific and unrealistic assumptions, and provide negligible predictive value. 

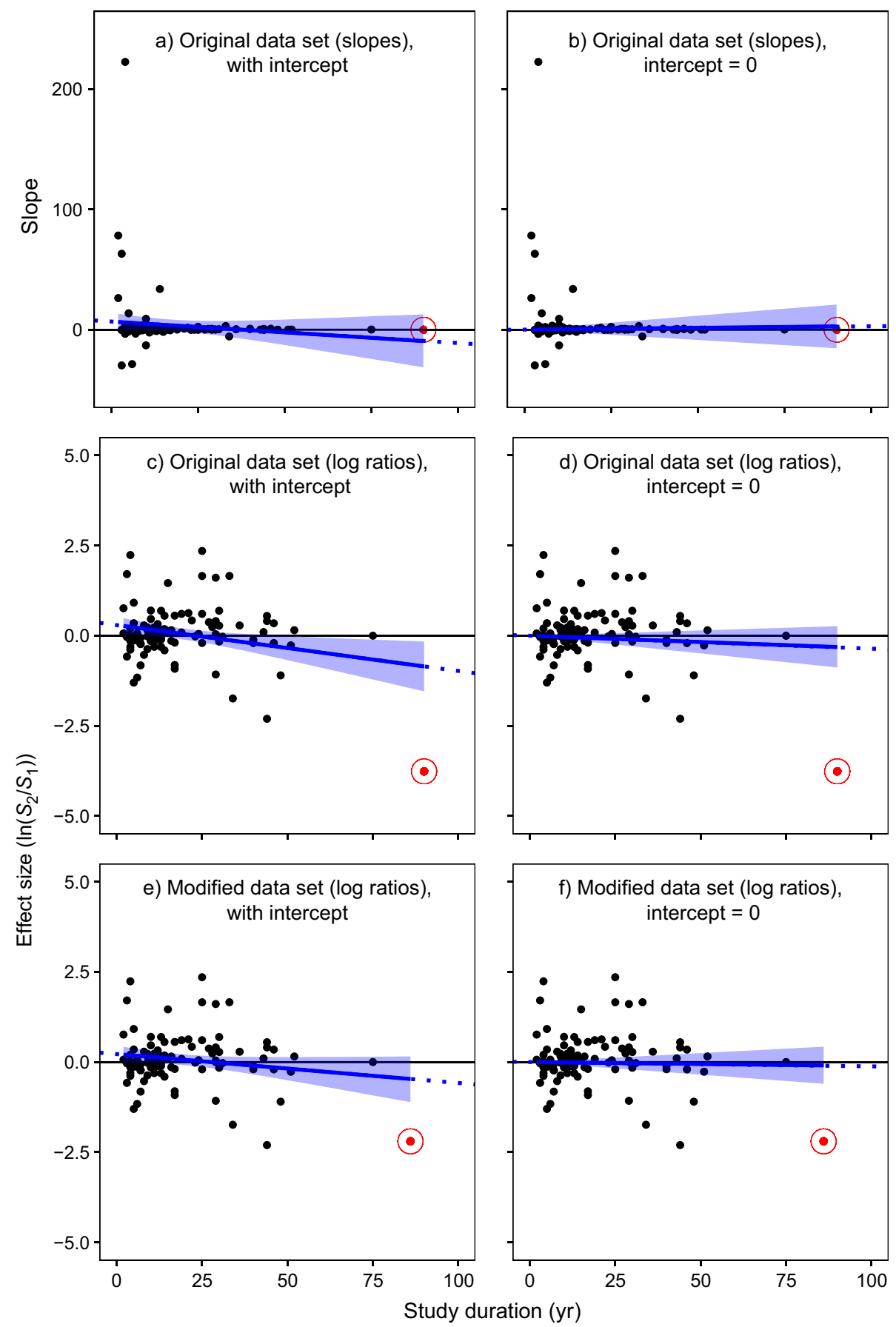

FIG. 3. Relationships between species richness change over time ( $y$-axis) and the duration of a study, using data from Dornelas et al. (2014). Relationships were modeled assuming either (a, c, e) a non-zero $y$-intercept or (b, e, f) a zero $y$-intercept, using either slopes $(\mathrm{a}, \mathrm{b})$ or log ratios (c-f) to express temporal biodiversity change, and either including one outlier (a-d; original data set) or not (e and f; modified data set). Lines represent the estimated effect size with credible intervals. See Appendix S1 for statistical methods and Metadata S1 for all data and computer code. [Color figure can be viewed at wileyonlinelibrary.com]

Whether using the realistic assumption of zero biodiversity change at duration $=0$, using a larger data set, taking account of an outlier, or analyzing slopes instead of $\log$ ratios, we find no convincing evidence that estimates of biodiversity change depend on study duration. In any given time series, it is clearly possible (and indeed likely) that trend detection will depend on the particular period of time analyzed. In our analyses, the 
observed trends were evenly spread above and below zero for the range of durations, and well-populated with data ( $<50 \mathrm{yr}$ or so). There is thus, at present, no evidence to support Gonzalez et al.'s conclusion that longer-duration studies systematically show average local biodiversity declines. We recognize that all of these analyses were carried out with respect to baselines determined by the beginning of the time series involved. There will be cases where ecosystems have lost or gained biodiversity before these observations began, but at present we cannot assess the frequency of these different scenarios.

\section{EFFects of Disturbance}

Another concern of Gonzalez et al. (see also Eisenhauer et al. 2016) was the simultaneous inclusion of (1) studies that characterize the effects of disturbance ("impacts") and (2) studies that characterize recovery from disturbance ("recovery"). If our goal had been to assess only the direct effects of disturbance (e.g., Supp and Ernest 2014), this would indeed have been inappropriate. But that was not the goal of either original paper. In a world with fire, grazing, logging, and other disturbances of varying intensity and frequency, to include only the effects of such disturbances and to ignore locations recovering from past disturbances constitutes the introduction of a bias in itself. Gonzalez et al. pursued this line of inquiry by selecting studies classified as "postdisturbance," "post-fire," and "cessation of grazing," for elimination from the Vellend et al. (2013) data set (i.e., leaving the impact-only data subset).

Unlike the primary analyses in Vellend et al. (2013), Gonzalez et al. analyzed raw log ratios rather than those expressed as change per decade, and they weighted studies by the square root of sample size. The result was a distribution of effect sizes with an upper confidence limit that was slightly negative ( $1 \%$ loss $)$. However, this significant result depends on the combined influence of the two analysis modifications introduced by Gonzalez et al. Using the original unweighted analyses and only the data reported in Vellend et al. (2013), the 95\% credible intervals for the impact-only data subset selected by Gonzalez et al. overlap zero both for raw log ratios, if only slightly ([-0.095, 0.012] or between $9 \%$ loss and $1 \%$ gain), and for change expressed per decade $([-0.061,0.031])$. More importantly, using the larger, updated data set, the upper credible intervals are well above zero (raw log ratio, $[-0.053,0.034]$; per decade, $[-0.038,0.032])$, allowing us to reject the conclusion that eliminating "recovery" studies reveals an average decline in local plant biodiversity.

More generally, we disagree with the argument that disturbances (anthropogenic or otherwise) are generally expected to cause a decline in local biodiversity. Empirical studies testing the effect of disturbance on species diversity find a wide range of results, including no effects, positive effects, negative effects, and hump-shaped relationships
(Mackey and Currie 2001, Supp and Ernest 2014, Newbold et al. 2015, Vellend et al. 2017). Even logging, implicitly emphasized in Gonzalez et al.'s analysis of forest cover change, is often followed by a short-term increase in local plant diversity (due to colonization of early-successional species) and a subsequent longer-term decline back to levels similar to old-growth forest (Halpern and Spies 1995, Duguid and Ashton 2013). In this scenario, capturing only the long-term "recovery" phase would bias results against positive trends rather than negative trends. The opposite scenario (the concern of Gonzalez et al.) certainly exists as well, with biodiversity potentially decreasing after disturbance. Disturbance does not have a unidirectional effect with a single recovery trajectory, so even if studies captured some phases of the disturbance cycle more than others, the consequences would not be easily predictable.

In sum, both short-term and longer-term changes in local biodiversity caused by disturbance are relevant to understanding temporal biodiversity trends, and the empirical data indicate highly context dependent effects of disturbance on the magnitude and direction of biodiversity change.

\section{The Ecological Literature is Indeed GeographicAlly Biased}

Ecological studies of all kinds have been conducted far more often in Europe and North America, and nearby waters, than elsewhere. In the case of our meta-analyses, we are unable at present to estimate with confidence how local biodiversity has changed in under-recorded or unrecorded regions, such parts of Africa or the Indian Ocean. This is a challenge for global analysis of biodiversity change, and we hope that highlighting this challenge will instigate more data collection in these regions. However, while any given subset of data might deviate slightly from the overall pattern, there was no obvious signal that geographic bias led to bias against finding biodiversity decline. For example, in Vellend et al. (2013), the estimated mean log ratios of species richness change over time for South America $(N=12)$, Asia $(N=9)$, Australia $(N=5)$, and Africa $(N=2)$ were all positive. One could choose to conduct an analysis giving greater weight to these understudied regions: this would shift the estimated central tendency toward biodiversity increases rather than decreases. In addition, the analyses of Gonzalez et al. show that one of the original meta-analyses had an underrepresentation of places with high recent human impacts (Vellend et al. 2013), while the other had an overrepresentation of places with high human impacts (Dornelas et al. 2014), and yet both studies show a similar distribution of temporal changes in local diversity.

Gonzalez et al. have identified some important axes along which we might improve the future representativeness of biodiversity studies (e.g., regional diversity, 
human impacts), but ultimately only new data from underrepresented regions can speak directly to what is happening in those parts of the world, and thus prompt a potential reassessment of conclusions. Local biodiversity change is very much dependent on specific, local circumstances, and new and interesting results from poorly known regions may well emerge in the future. Improving the spatial representation of these regions is a high priority in obtaining better estimates of local biodiversity change.

In sum, Gonzalez et al. present analyses to demonstrate a point noted in both original papers: the data are geographically biased. Precisely the same limitation applies to most ecological synthesis and meta-analysis papers (e.g., Cardinale et al. 2012, Hooper et al. 2012, Elahi et al. 2015, Haddad et al. 2015), in which there was no such vigorous effort to quantify geographic bias and its attendant consequences for limiting the scope of conclusions. We are working with the best data available, and continue to assemble data, to directly document temporal biodiversity change at the local scale. Converting natural ecosystems to croplands or parking lots causes a local loss of biodiversity (Newbold et al. 2015), but otherwise there is a great deal of variation but no clear tendency for the net temporal local biodiversity trend to be different from zero across the sites in the available data (Vellend et al. 2013, Dornelas et al. 2014, Elahi et al. 2015).

\section{To Conclude}

We agree with Gonzalez et al. concerning the need for better biodiversity monitoring in the future. Our knowledge of a great many places on earth is quite limited, and many drivers of biodiversity change are expected to push in opposite directions (Vellend et al. 2017). For example, nonnative species introductions typically increase regional-scale species richness (Sax and Gaines 2003, Winter et al. 2009), and in areas that are currently cold and humid (e.g., temperate-zone mountain tops), species richness is also expected to increase due to climate warming (Pauli et al. 2012). On the other hand, nitrogen deposition often causes plant diversity to decline (Simkin et al. 2016), and for some taxa habitat fragmentation can do the same (Haddad et al. 2015). How different forces balance out in the future can best be determined by systematic, long-term monitoring - a major priority for future research in ecology and conservation.

Causes and trends of local biodiversity, and therefore any applied consequences, are just as described: local. The global average across many local trends is thus of applied significance only indirectly, via framing arguments about the consequences of biodiversity change (e.g., Hooper et al. 2012). Given the data at hand, we can reject the notion of local biodiversity loss as the general rule, and whether new data reveal a ratio of positive vs. negative trends at 50:50, 60:40 (positive mean), or 40:60 (negative mean), context dependence and site-specificity would remain the dominant pattern. The most generally applicable statement we can make at present is that in most situations we expect substantial changes in species composition, that is, species turnover, with important implications for biodiversity conservation efforts (Dornelas et al. 2014, Magurran 2016).

\section{ACKNOWLedgments}

We would like to thank three anonymous reviewers for insightful comments and suggestions that helped improve the manuscript.

\section{Literature Cited}

Cardinale, B. J., et al. 2012. Biodiversity loss and its impact on humanity. Nature 486:59-67.

Colwell, R. K., and D. C. Lees. 2000. The mid-domain effect: geometric constraints on the geography of species richness. Trends in Ecology \& Evolution 15:70-76.

Dornelas, M., N. J. Gotelli, B. McGill, H. Shimadzu, F. Moyes, C. Sievers, and A. E. Magurran. 2014. Assemblage time series reveal biodiversity change but not systematic loss. Science 344:296-299.

Duguid, M. C., and M. S. Ashton. 2013. A meta-analysis of the effect of forest management for timber on understory plant species diversity in temperate forests. Forest Ecology and Management 303:81-90.

Eisenhauer, N., et al. 2016. Biodiversity-ecosystem function experiments reveal the mechanisms underlying the consequences of biodiversity change in real world ecosystems. Journal of Vegetation Science 27:1061-1070.

Elahi, R., M. I. O’Connor, J. E. Byrnes, J. Dunic, B. K. Eriksson, M. J. Hensel, and P. J. Kearns. 2015. Recent trends in local-scale marine biodiversity reflect community structure and human impacts. Current Biology 25:1938-1943.

Gonzalez, A., B. J. Cardinale, G. R. H. Allington, J. E. Byrnes, K. A. Endsley, D. G. Brown, D. U. Hooper, F. Isbell, M. I. O'Connor, and M. Loreau. 2016. Estimating local biodiversity change: a critique of papers claiming no net loss of local diversity. Ecology 97:1949-1960.

Haddad, N. M., L. A. Brudvig, J. Clobert, K. F. Davies, A. Gonzalez, R. D. Holt, T. E. Lovejoy, J. O. Sexton, M. P. Austin, and C. D. Collins. 2015. Habitat fragmentation and its lasting impact on Earth's ecosystems. Science Advances 1: e1500052.

Halpern, C. B., and T. A. Spies. 1995. Plant species diversity in natural and managed forests of the Pacific Northwest. Ecological Applications 5:913-934.

Hooper, D. U., E. C. Adair, B. J. Cardinale, J. E. K. Byrnes, B. A. Hungate, K. L. Matulich, A. Gonzalez, J. E. Duffy, L. Gamfeldt, and M. I. O'Connor. 2012. A global synthesis reveals biodiversity loss as a major driver of ecosystem change. Nature 486:105-108.

Mackey, R. L., and D. J. Currie. 2001. The diversity-disturbance relationship: Is it generally strong and peaked? Ecology 82:3479-3492.

Magurran, A. E. 2016. How ecosystems change. Science 351:448-449.

Newbold, T., L. N. Hudson, S. L. Hill, S. Contu, I. Lysenko, R. A. Senior, L. Börger, D. J. Bennett, A. Choimes, and 
B. Collen. 2015. Global effects of land use on local terrestrial biodiversity. Nature 520:45-50.

Pauli, H., et al. 2012. Recent plant diversity changes on Europe's mountain summits. Science 336:353-355.

Sax, D. F., and S. D. Gaines. 2003. Species diversity: from global decreases to local increases. Trends in Ecology \& Evolution 18:561-566.

Simkin, S. M., E. B. Allen, W. D. Bowman, C. M. Clark, J. Belnap, M. L. Brooks, B. S. Cade, S. L. Collins, L. H. Geiser, and F. S. Gilliam. 2016. Conditional vulnerability of plant diversity to atmospheric nitrogen deposition across the United States. Proceedings of the National Academy of Sciences USA 113:4086-4091.

Supp, S. R., and S. K. Ernest. 2014. Species-level and community-level responses to disturbance: a cross-community analysis. Ecology 95:1717-1723.
Vellend, M., L. Baeten, A. Becker-Scarpitta, V. BoucherLalonde, J. L. McCune, J. Messier, I. H. Myers-Smith, and D. F. Sax. 2017. Plant biodiversity change across scales during the Anthropocene. Annual Review of Plant Biology 68 , in press.

Vellend, M., L. Baeten, I. H. Myers-Smith, S. C. Elmendorf, R. Beauséjour, C. D. Brown, P. De Frenne, K. Verheyen, and S. Wipf. 2013. Global meta-analysis reveals no net change in local-scale plant biodiversity over time. Proceedings of the National Academy of Sciences USA 110:19456-19459.

Winter, M., O. Schweiger, S. Klotz, W. Nentwig, P. Andriopoulos, M. Arianoutsou, C. Basnou, P. Delipetrou, V. Didžiulis, and M. Hejda. 2009. Plant extinctions and introductions lead to phylogenetic and taxonomic homogenization of the European flora. Proceedings of the National Academy of Sciences USA 106:21721-21725.

\section{SUPPORTING INFORMATION}

Additional supporting information may be found in the online version of this article at http://onlinelibrary.wiley.com/ doi/10.1002/ecy.1660/suppinfo 